\title{
ARTIFICIAL INTELLIGENCE AND ITS ROLE IN HUMAN RESOURCE MANAGEMENT
}

\section{SHIVANI TIWARI}

Research Scholar, Department of Management, Glocal University, Saharanpur, Uttar Pradesh, India \begin{abstract}
It is the development and research of creating intelligent computers, in fact intelligent computer programs. This is synonymous with the traditional challenge to use computers to comprehend human intelligence, but AI should not be restricted to approaches that are scientifically detectable. The use of algorithms to perform tasks that typically involve the knowledge of humans implies artificial intelligence. It implies that algorithms are generated for the description, interpretation and drawing of data predictions. This also includes system maintenance, new application development and progress over time. Human Resource Management (HRM or HR) is the systematic approach to handling employees efficiently within an enterprise or organisation so that they can help a business achieve a competitive edge. This is designed to optimize the efficiency of workers in relation to the corporate goals of an organization. This is the business department which cares for the recruiting, management and firing of employees. This further reflects on the position of staff within the organization to insure that common standards are still in effect. Nevertheless, the main worry of the world's populace is how AI shows its effect on work growth through diverse sectors worldwide. The reality is that it is not emerging technology that completely eliminates people; the roles of AI can affect a large percentage of workers, and the HR executives and organisations have a responsibility to focus on their workforce demands and future results. In the end, most organizations are increasingly using AI-related approaches for hiring on the basis of research but AI will be in HR anywhere in the near future: whilst recruitment, preparation, boarding, productivity review, retaining, etc. may occur, the majority of organisations, due to their deployment costs, often lag behind in incorporating AI into their HR activities. But how people can adapt and interpret these innovations in order to build wealth and stability is all about here.

KEYWORDS: Artificial Intelligence, Human Resource, HR Technology, Human Resource Management, Machine Learning
\end{abstract}

Received: Jun 08, 2020; Accepted: Jun 28, 2020; Published: Sep 08, 2020; Paper Id.: IJMPERDJUN20201109

\section{INTRODUCTION}

Artificial Intelligence (AI) is defined as an ideal cognitive machine which is a dynamic agent that understands the world and takes action and for some reason maximizes its chances of success.Every day, the term artificial intelligence takes on new characteristics. The word Science and Technology of Intelligent Machinery was first used by John McCarthy.It is a computer-demonstrated intelligence, as opposed to the natural intellect of humans. Artificial intelligence (AI)[1] refers to technology that is capable of executing operations that require a certain amount of intellectual ability that is, a machine capable of achieving what a human being would do in other words, a tool trained to do what a human can do. It is useful in many different corporate roles where it can help to reduce the load on employees at the workplace. Rapid business changes need quick response.Growing business success is based on the productivity with which it intelligently integrates personnel, strategies and equipment to obtain a minimum transition benefit. 
Artificial Intelligence (AI) has been a dream for decades, and the writers of science fiction have visualized the development of sophisticated robots that could carry out human activities or even succeed in other fields. Artificial Intelligence ( $\mathrm{AI}$ ) is the colouring source for fantasy pictures during the period of digitisation [2].It is a possible turning point that transforms our life both at home and at work. The Artificial Intelligence Department (AI) operates in a range of ways as an intelligent assistant to the user, such as the Amazon's Alexa, delivering information, monitoring lighting, locking and unlocking intelligence homes, etc.

Nowadays, artificial intelligence has penetrated the overall structure of the enterprise and one of the fields is the Human Resources Division, which uses the AI human system to replace person and other roles in the Human Resources Department, such as applicant selection, recruiting, coordination of human resource operations and success improvement, etc. Humans and intelligent algorithms work together to generate an ever-increasing volume of HR data in the cloud, and the application of artificial intelligence analyzes give a deeper perspective into how to function and run. AI can help to effectively simplify a variety of back-office tasks for effective HR transfers and service delivery[3], [4].

The AI is distinguished from the normal applications by three main elements, high speed computation, accurate data and sophisticated algorithms in significant numbers. Key AI systems improve the precision and reliability of routine operations with an algorithm that connects data quality with fast computing resources.

Human resources are generally viewed as one of the most valuable assets of any enterprise and, as a result, handling this asset effectively is deemed to be a core managerial responsibility.Managing human resources includes a broad variety of specific activities, including recruiting, workplace success monitoring, professional training and job growth, and employee contribution benefits.Any organization's success depends on how well it intelligently combines personnel, processes and equipment to deliver change efficiency at reduced expense.

Artificial intelligence is the basis for a new age of digital transformation between diverse channels through the use of many AIs. Tools for HRM, including recruiting, placement, training and development, performance administration, benefits and reward management. Through changing existing business systems and making it easier for the task force to be more competitive with artificial intelligence, artificial technology enhances intellectual resources. This not only streamlined human thinking, but has introduced strong company development and has proven incredibly useful in enhancing workplace relationships and in increasing employee satisfaction.

Improving market efficiency is the main objective of most businesses. Innovation in discovering new technologies and methods to build better systems is a constant problem in today's economy. Enhancing human behaviour has led to a new increase in the use of AI robotics. Computers and software has been a must for today's operations. HR technology can be described as any methodology for the recruiting, utilization, preservation and management of human resources, assistance with HR and outsourcing of HR. HR technology has been named [5]-[7]. The small, medium-scale and big corporations slowly employ HR technologies to satisfy their customers ' demands.

In this article, we explore how improvements should be made to the contribution to HRm and AI 's understanding [8]-[10].

\section{We Identify Four Problems Surrounding the Application of Data Analytics Technologies in Human Resources Practices:}

- The nature of HR processes, 
- The limitations imposed by limited data sets,

- Ethical concerns relating to justice and legal restrictions, and

- Staff responses of network engineering operations.

The research author (Duchessi, O'Keefe, \& O'Leary, 1993) addressed the effect of artificial intelligence and emerging technologies on control and accountability for decision-making, cost savings and improved efficiency, staffing changes and downsizing on corporate structure, staff management.

\subsection{Research Questions}

- How does Artificial Intelligence act?

- Why does AI vary from ordinary software?

- What role does it plays in HRM?

\section{REVIEW OF LITERATURE}

Geetha R \&BhanuSree Reddy D, "Artificial Intelligence Recruitment: Conceptual study"this paper's main objective is to study how Artificial Intelligence influences the recruitment strategy. The report further sheds light on the methods used when recruitment by AI firms. This research is largely undertaken on the basis of secondary knowledge sources such as philosophical documents, numerous peer reviewed journal articles, books, and websites are used to further develop the idea. Secondary sources such as blogs, journals, studies, technical writing, and books are referred for the full paper to be drawn up.- Combining human and AI helps in computer management, saving organisations money and resources for better precision and transparency in the overall recruiting process.

Merlin \&Jayam, "Management of human resources by artificial intelligence"- Simple, and Applied Mathematics International Journal. This paper attempts to address the possibilities of how Artificial Intelligence transforms and supports functions of the Human Resource such as recruitment, training, and talent management.Use real-time examples, retention offers insights into the intersection of artificial intelligence \& human resources and addresses potential effects on HR employees in the end.They used secondary analysis to investigate the ways in which Artificial Intelligence enhances and facilitates human resource roles such as recruiting, preparation, talent acquisition and retention by real-time examples, offers insights into the convergence of Artificial Intelligence \&Cases in human resource management and ultimately discussing the potential impact on the HR workforce.

SriramMalathi, and L. Exploring "Gandhi, Shri DharmasthalaManjunatheshwara Dynamic Value of Machine Learning ( ML) in Human Resource Management — A Vital Study of the IT Industry "This paper focuses on the use of machine learning that has replaced certain human resource management roles, especially in the IT field.To use a model that is based on the results. In this paper a few organizations' cases were chosen to demonstrate how they changed their HR processes using Machine Learning. Example cases are taken to illustrate the success of the organizations in applying Machine Learning in HR. There can be many innovative ways of applying Machine Learning and Artificial Intelligence to HR functions in conclusion.

Ian Bailie Head of HR-" An Analysis of Artificial Intelligence and its Effect on Human Resources "This article talks of major companies embracing AI and exploring the fundamentals of AI and how AI is being 
implemented in HR. It's been built for those wishing to learn more about the potential application of AI in HR.This explores both industry and academic outlets to establish a description of AI in the Indian This Sector Artificial Intelligence \& Human Resource Management, and its use in market with a particular emphasis on HR. The study also includes key data found in Cognition's AIpowered HR Services Database, survey findings, and company and expert interviews.It focused on the few budget questions to implement new AI tools, technology sufficiently advanced from prospective ROI, HR having the right technical expertise to work with AI and considering ethical implications when implementing AI technology.

Shweta Jain-The Engine Driving the Next Wave of Innovation in Industry, the author explores in this paper how artificial intelligence helps in complete digital transformation when the company integrates well with the different units such as HR, marketing, accounting, manufacturing or procedure.The author concluded in his research, which included recruiting, placement, planning, growth, results, awards and reward management, that HR practitioners should use different AI technologies and resources for all HR functions.

For Dianna L. And. Discuss emerging technical pressures on HR procedures and address the benefits and possible disadvantages of using information technology. The authors suggested that the trend against her is likely to grow in the future, but many of the typical results of HR research still extend to HR.

Buzko et al. find that the key factor affecting the amount of training in the business is the organization's net profit for the previous year and the transition from a single information management model to a continuous model makes for quicker and more precise adaptation of environmental requirements. The writers have argued that using artificial intelligence technology for decision making is becoming more important in the current market conditions.

Sander Kloppenburg and Robert Charlier, PwC, Artificial Intelligence in HR: A Nobrainer-It is a big point in today's company to pursue the best talent at low cost and with less time!According to this article, which was based on analysis by PwC's global network after the different facets of artificial intelligence, the input of business associates, interviews with industry experts, and insightful remarks from the participants of our round Table event, arranged in collaboration with Seed Connection.

\section{METHODS}

The research study uses a descriptive research design. In this research used secondary data in the research analysis. Secondary data is obtained from academic papers, written articles, internet directories, HR forums, and survey results released by numerous polling organizations.

\subsection{Design:}

\subsubsection{Questionnaire:}

The main data gathered for this analysis is this article. According to the survey monkey website, the sample size for up to 3,000 Romandes HR members is 310 with a 5\% margin variation for a population of 3,000.

The questions for the survey were built for the 5-point Likert scale. The study was based on a theoretical basis that the authors presented and was similar to other research studies. The survey has been divided into seven sections: HR process, impact analysis, issues and challenges, AI ability, focus recruiting, data processing, market orientation and demographic information. 


\subsection{Sample:}

\subsubsection{Focus and Selection:}

Around June and July 2019, the authors' committee published a survey of members of the HR Association Romandes Section. The company communicated this to the Group's HR divisions.A total of 541 participants replied and 310 were taken into account after being tested for qualifications. The sample had a total of 72 questions. Extensive work on existing literature and topic details.Information in AI and organizational behaviour is obtained under the basis that AI in HRM intersects with organizational behaviour.

\subsubsection{Instruments:}

\section{Two Statistical Methods Were Used to Analyze the Outcome of the Survey:}

Descriptive mathematical research to analyze and understand the general situation, intentions and aspirations of AI in the HRM.

Hypothesis research protocols (t-tests, Chi Square and ANOVA) for the evaluation of the statistical associations between such predictor variables as market focus, planning and adaptation of AIs, including their potential importance and consideration for the impact of AI in the field of HRM work relief and apprehension of job cuts, both now and in the future.

\subsection{Data Analysis:}

The research tool was chosen for analyzing the results. This methodology was selected based on the original framework of analysis in interpretativism, since thematic are a appropriate approach for this framework. This is focused on the elimination of qualitative data by labelling, for example, the interviews performed and the recognition of new topics. In general, a themed structure is very flexible and hence appropriate for other fields of study. While the key points of the data analyzes, the topics found by the thematic study are contrasting with both the theoretical context and model chosen.The details from the interviews were first transcribed, interpreted and read multiple times in a written form. Original codes from the collected data are generated in the second stage. The pre-set codes included traditional recruitment, artificial intelligent technology, performance, time management, automation, robots, skill creation, and personnel. Remedies, attributes, decision, connectivity, screening and ability were other codes which emerged during data analysis. In the third step, the codes found were closely studied and explored how to incorporate them in a pattern. The authors began to elaborate established themes in the fourth phase, that is, the themes analysis.

\section{RESULTS}

\subsection{Applications for Artificial Intelligence in Human Resources Management:}

The use of HRM AI involves the transition of people's work to an external agent. The purpose and complementary application of $\mathrm{AI}$ is to improve and to strengthen the capacity of human users to accomplish their objectives by leveraging the powers of certain actors with respect to the use of AI to promote pro-social engagement. Throughout the future of AI and its potential to be incorporated into HRM systems for the next five years, the adoption and deployment of AI in the HRM industry is an important aspect in determining standards.

We consider a major gap in the cross-analysis of the findings of these two claims. This leads one to conclude that the more they concluded that AI is widely used in hrm processes over the next five years, as they replied Yes to having already implements and embarks on AI in their HRM processes.

Survey results indicate that $50 \%$ of respondents "partly believe" that AI is widely used in HRM applications over the next five years. The survey has assessed and estimated the number of employees who use AI in their HRM systems. at 
the same time. $31 \%$ answered yes. Historically, HR staff leaders, workers, or supervisors may have had to carry out these duties. Instead, the introduction of these AI-based approaches permitted the reorganization of HRM processes to reduce the workload of employees.

\section{DISCUSSIONS}

This research indicates a connection between the HRM acceptance of AI and their statistical importance. This connection indicates that the embrace of new technologies is also a indication that a organization is able to take chances and is open to creativity. Yes, all of these variables are used in the calculation of HRM.

Based on both theory and individual literature, HR contains a plurality of principles from many scholarly areas.Taking Miller and Friesen as a starting point, three dimensions of creative attitude were often defined and introduced in research: creativity, proactiveness and risk-taking. According to this typology, the characteristics of this study, including creative focus in contrast with the implementation of $\mathrm{AI}$ in human resources, were thus divided into three classes.

The creative orientation of an organization denotes its internal structures, behaviour, strategies, procedures, practices and decision-making.

\section{CONCLUSIONS}

Financial stocks are at times competitive or unpredictable. Talent depletion is a reality every day. Workweeks can be required to reach 60 hours on a daily basis. Management would now be more effective and creative. There has been a suggestion that, while there is much uncertainty about what is to come with AI, AI's implementation and delivery is a constant necessity for longevity. AI is a mechanism that optimizes operations through organizations. AI anticipates shifts and developments in human capital.

The analysis discussed HRM hypotheses by members of the HR Romandes committee on AI perception. Through a survey which assessed the perceived value of AI in HRM, including its risks, shortcomings and preparations for its execution, we found the acceptance of AI in HRM generally positive.Therefore, there is little risk of a negative impact on the number of job cuts. Throughout the report, the members of the Romands Subcommittee on AI Intelligence discussed HRM theories. In a study assessing the perceived importance of AI in HRM, including its threats, drawbacks and plans for its deployment, we found broadly strong approval of AI in HRM.The assertion, "The use of AI is an advantage for the company," led to the following result: 30 per cent strongly agreed, 8 per cent partially agreed, 18 per cent neutral, 4 per cent partially disagreed and 1 per cent s4trongly disagreed. According to the assertion, "Companies that incorporate AI would have a strategic edge in the industry," 13 per cent strongly supported, 50 per cent partially approved, 20 per cent were indifferent, 12 per cent were slightly disappointed and 2 per cent strongly objected.

Based on our results, we conclude that the AI systems should be properly analyzed and implemented. In order to prevent work cuts and uncertainty, organizations should ensure adequate program knowledge and transition of skills to HR staff.

\section{REFERENCES}

1. V. Scotti, “Artificial intelligence,” IEEE Instrum. Meas. Mag., 2020.

2. Bansla and N. Bansla, “Artificial intelligence,” Int. J. Appl. Eng. Res., 2012.

3. K. Upadhyay and K. Khandelwal, “Applying artificial intelligence: implications for recruitment,” Strateg. HR Rev., 2018. 
4. R. Charlier and S. Kloppenburg, “Artificial Intelligence in HR: a No-brainer,” Pwc, 2017.

5. P. Reilly, “The Impact of Artificial Intelligence on HR Function,” Inst. Employ. Stud., 2018.

6. jeanne Meister, “Ten HR Trends In The Age Of Artificial Intelligence,” Forbes. 2019.

7. T. Cohen, "How to leverage artificial intelligence to meet your diversity goals," Strateg. HR Rev., 2019.

8. R. Kramar, "Beyond strategic human resource management: Is sustainable human resource management the next approach?,” Int. J. Hum. Resour. Manag., 2014.

9. P. Thompson, “The trouble with HRM,” Hum. Resour. Manag. J., 2011.

10. N. Bloom and J. Van Reenen, Human resource management and productivity. 2011. 

